\title{
Short QT Syndrome: A Comprehensive Genetic Interpretation and Clinical Translation of Rare Variants
}

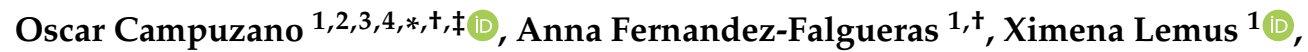 \\ Georgia Sarquella-Brugada ${ }^{3,5}$, Sergi Cesar ${ }^{5}{ }^{1}$, Monica Coll ${ }^{1}$, Jesus Mates ${ }^{1}$, Elena Arbelo ${ }^{2,6}$, \\ Paloma Jordà ${ }^{2,6}$, Alexandra Perez-Serra ${ }^{1}$, Bernat del Olmo ${ }^{1}$, Carles Ferrer-Costa ${ }^{1}$, \\ Anna Iglesias ${ }^{1}$, Victoria Fiol ${ }^{5}$, Marta Puigmulé ${ }^{1}$, Laura Lopez ${ }^{1}$, Ferran Pico ${ }^{1}$, Josep Brugada ${ }^{2,5,6}$ \\ and Ramon Brugada $1,2,3,7, *, \ddagger$ (D)

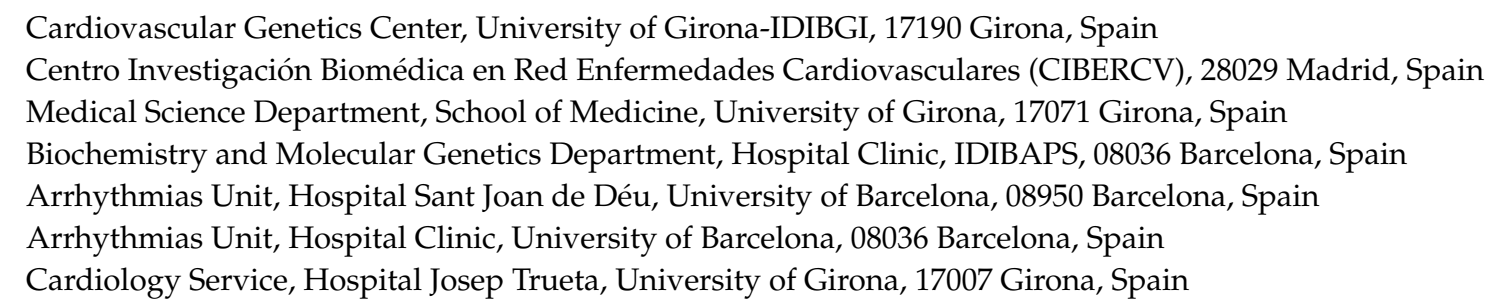

Received: 8 June 2019; Accepted: 11 July 2019; Published: 16 July 2019

\begin{abstract}
Short QT syndrome, one of the most lethal entities associated with sudden cardiac death, is a rare genetic disease characterized by short QT intervals detected by electrocardiogram. Several genetic variants are causally linked to the disease, but there has yet to be a comprehensive analysis of variants among patients with short QT syndrome. To fill this gap, we performed an exhaustive study of variants currently catalogued as deleterious in short QT syndrome according to the American College of Medical Genetics and Genomics and the Association for Molecular Pathology. Analysis of the 32 variants described in the literature determined that only nine $(28.12 \%)$ have a conclusive pathogenic role. All definitively pathogenic variants are located in KCNQ1, KCNH2, or KCNJ2; three genes encoding potassium channels. Other variants located in genes encoding calcium or sodium channels are associated with electrical alterations concomitant with shortened QT intervals but do not guarantee a diagnosis of short QT syndrome. We recommend caution regarding previously reported variants classified as pathogenic. An exhaustive re-analysis is necessary to clarify the role of each variant before routinely translating genetic findings to the clinical setting.
\end{abstract}

Keywords: short QT syndrome; sudden cardiac death; genetics; pathogenicity

\section{Introduction}

In 2000, Gussak et al. described short QT syndrome (SQTS) as a new clinical entity. Further, it was described as inherited condition because four individuals from the same family presented with idiopathic and persistent short QT intervals via electrocardiogram (ECG) [1]. In 2011, Gollob et al. proposed a SQTS diagnostic criteria based on four components including ECG, clinical history, family history, and genotype [2]. In 2013, a consensus established that a cutoff value of QTc $\leq 330 \mathrm{~ms}$ should be used for the diagnosis [3]. Currently, clinical diagnosis of SQTS is characterized by a shortened QT interval (QTc $<340 \mathrm{~ms}$ ), tall and peaked T waves, and poor rate adaptation of the QT interval, all 
without structural heart abnormalities [4]. In addition, SQTS can be also diagnosed with a QTc interval of $</=360 \mathrm{~ms}$ with one or more of: (a) a confirmed pathogenic mutation; (b) a family history of SQTS (c) a family history of sudden death at age $<40$ years, and (d) survival from a VT/VF episode in the absence of structural heart disease [4].

Individuals with SQTS can exhibit widely varying symptoms, from never experiencing health problems associated with the disease to dizziness, syncope, and even sudden cardiac death (SCD) due to ventricular fibrillation. This malignant arrhythmia can occur any time from early infancy to old age, although most cases occur at a young age. There is also a male predominance to SQTS [5]. The prevalence of SQTS is estimated at $0.02-0.1 \%$; however, this may be underestimated due to no more than 100 cases have been diagnosed worldwide to date [6]. Applying different QTc correction formulae leads to significant reclassification in SQTS probability. The risk of SCD among SQTS patients is high and an implantable cardioverter defibrillator (ICD) may be used to prevent SCD [4]. However, SQTS may be relatively benign if suitable risk assessment and individualized pharmacological measures are applied with early detection [7].

In 2004, Brugada et al. identified the first genetic variant associated with SQTS, located in the $\mathrm{KCNH} 2$ gene [8]. Additional potentially pathogenic variants have been reported in five genes (CACNA2D1, KCNH2, KCNJ2, KCNQ1, and SLC4A3); all exhibit autosomal dominant inheritance. Comprehensive genetic analysis is used to identify the cause of SQTS in nearly $20 \%$ of diagnosed cases. However, this percentage may be misleading due to the low number of reported families. This lack of families also impedes a robust genotype-phenotype correlation assessment for risk stratification [4]. Genetic variants have been reported in three other genes: $C A C N A 1 C, C A C N B 2 b$, and $S C N 5 A$, although individuals with these variants show a characteristic phenotype of Brugada syndrome (BrS) with concomitant shortened QT intervals, but without a conclusive clinical diagnosis of SQTS. Despite controversial data, current guidelines recommend genetic analysis of five genes: $K C N H 2, K C N Q 1$, $K C N J 2, C A C N A 1 C$, and CACNB2b) in the diagnosis of SQTS [4]. Family assessment is mandatory when a patient is diagnosed via genetic testing, allowing for the early identification of relatives at risk [9].

In 2015, the American College of Medical Genetics and Genomics and the Association for Molecular Pathology (ACMG/AMP) published recommendations for the interpretation of rare variants, hoping to clarify their pathogenic role [10]. Each entry describes evidence for both pathogenicity and benignity to enable the classification of variants as pathogenic, likely pathogenic, variants of uncertain significance, likely benign, and benign. The Human Genome Mutation Database (HGMD) classifies a few variants as disease-causing mutations and potentially damaging associated with SQTS, although these variants have not been comprehensively examined following ACMG/AMP recommendations. However, variants' pathogenicity classifications are based on limited evidence. Therefore, we performed a comprehensive analysis of all published data to clarify the roles of rare variants currently reported as potentially deleterious in SQTS and to establish the genes associated with conclusive clinical diagnosis of SQTS.

\section{Experimental Section}

Data concerning "Short QT syndrome and/or Short QT interval" was collected from: HGMD (http://www.hgmd.org), ClinVar (https://www.ncbi.nlm.nih.gov/clinvar/intro/), the National Center for Biotechnology Information SNP database (http://www.ncbi.nlm.nih.gov/SNP), Index Copernicus (http://en.indexcopernicus.com), Google Scholar (https://scholar.google.es), Springer Link (https: //link.springer.com), Science Direct (https://www.sciencedirect.com), Excerpta Medica Database (https: //www.elsevier.com/solutions/embase-biomedical-research), and the IEEE Xplore Digital Library (http://ieeexplore.ieee.org/Xplore/home.jsp).

Genetic variants identified in articles were contrasted with variant data from HapMap (http: //hapmap.ncbi.nlm.nih.gov), 1000 Genomes Project (http://www.1000genomes.org), Exome Variant Server -EVS- (http://evs.gs.washington.edu/EVS), Genome Aggregation Database -gnomAD- (http: //gnomad.broadinstitute.org/), and Exome Aggregation Consortium -ExAC- (http://exac.broadinstitute. 
org), including recently added data concerning copy number variations (CNV). Concerning global frequencies in population databases, it is important to remark that ethnic differences should be taking into account in a final classification. In addition, we consulted data concerning amino acid structure, including Grantham score and MutPred (http://mutpred.mutdb.org). An exhaustive in silico prediction of pathogenicity of genetic variations also was assessed using Combined Annotation Dependent Depletion -CADD- (http://cadd.gs.washington.edu), Genomic Evolutionary Rate Profiling -GERP- (http: //mendel.stanford.edu/SidowLab/downloads/gerp), Mendelian Clinically Applicable Pathogenicity Score -M-CAP- (http://bejerano.stanford.edu/mcap), Fathmm-MKL (http://fathmm.biocompute.org.uk/ fathmmMKL.htm), MetaSVM and MetaLR (which represents two ensemble methods that combine 10 predictor scores: SIFT, PolyPhen-2 HDIV, PolyPhen-2 HVAR, GERP, MutationTaster, Mutation Assessor, FATHMM, LRT, SiPhy, and PhyloP), Mutation Taster (http://www.mutationtaster.org), Mutation Assessor (http://mutationassessor.org), Phylogenetic Analysis -PhyloP- (http://compgen.cshl.edu/phast/ background.php), Protein Variation Effect Analyzer -PROVEAN- (http://provean.jcvi.org/index.php), and Polymorphism Phenotyping v2 -PolyPhen2, PPH2- (http://genetics.bwh.harvard.edu/pph2).

Variants were classified according to ACMG/AMP standards and guidelines for the interpretation of sequence variants (ACMG classification) [10]. Hence, ACMG/AMP recommendations describe several items of pathogenicity (PVS: Evidence of Pathogenicity Very Strong, PS: Evidence of Pathogenicity Strong, PM: Evidence of Pathogenicity Moderate, and PP: Evidence of Pathogenicity Supporting), and benignity (BA: Evidence of benign impact Stand-alone, BS: Evidence of benign impact Strong, and BP: Evidence of benign impact Supporting), enabling and a final score and consequent classification of variants in P: Pathogenic, LP: Likely Pathogenic, VUS: Variant of Uncertain Significance, LB: Likely Benign and B: Benign. The PM2 item in the ACMG classification was considered fulfilled if minor allele frequency (MAF) in relevant population databases was $\leq 0.1 \%$ [11]. Concerning frequency of disease-causing variants, vast majority of pathogenic variants are extremely rare $(<0.01 \%)$ [12]. Concerning PVS1, it should only be used for variants in genes where loss of function is a previously established disease mechanism (https://www.ncbi.nlm.nih.gov/projects/dbvar/clingen/) [13]. In addition, some items of ACMG/AMP may underlie a lack of specificity or ambiguous or contradictory interpretations, so we check the parameters using Sherloc (semiquantitative, hierarchical evidence-based rules for locus interpretation) [14]. Three authors independently conducted a comprehensive investigation of published data concerning each variant analyzed in our study. All investigators discussed data included in each item of the ACMG, and consensus a final classification of all variants in order to avoid any bias.

\section{Results}

Published data concerning SQTS show 15 variants reported as disease mutations (DM): two in $K C N Q 1$, eight in KCNH2, three in KCNJ2, and one variant in SLC4A3. In addition, 17 variants have been classified as having ambiguous significance due to a lack of conclusive data and conflicting clinical phenotypes. These rare variants have been identified in five genes: KCNQ1, KCNJ2, CACNA1C, $C A C N B 2$, and $S C N 5 A$. Therefore, rare variants potentially associated with SQTS or similar phenotypes are reported in eight genes: CACNA1C, CACNA2D1, CACNB2, KCNH2, KCNJ2, KCNQ1, SCN5A, and SLC4A3.

\subsection{The CACNA2D1 Gene}

Only one variant classified as pathogenic for SQTS -p.(Ser755Thr), rs151327713, CM111612- has been reported [15]. Other reports identified the same rare variant in a patient diagnosed with $\mathrm{BrS}$, and functional in vitro studies showed no significant ion current alterations in mutant cells in comparison to controls. The variant is now identified in low frequencies (Table 1). ClinVar classifies the variant as having ambiguous significance. In addition, in silico databases show a contradictory role for this variant (Table 2). Considering all data, p.S755T should be classified as Benign for SQTS following ACMG/AMP recommendations (Tables 1 and 3, Figure 1). 
Table 1. Pathogenic and additional variants associated with Short QT Syndrome.

\begin{tabular}{|c|c|c|c|c|c|c|c|c|}
\hline Gene & Protein & dbSNP & $\begin{array}{c}\text { EVS MAF (\%) } \\
\text { EA/AA/All }\end{array}$ & ExAC Alleles (\%) & gnomAD Alleles (\%) & HGMD (DM) & ClinVar & $\begin{array}{l}\text { ACMG/AMP } \\
\text { Classification }\end{array}$ \\
\hline \multirow{10}{*}{ CACNA1C } & p.(Ala39Val) & rs121912776 & - & - & - & $\mathrm{BrS}+\operatorname{stnQT}$ & $\mathrm{P}$ & VUS \\
\hline & p.(Gly490Arg) & rs121912775 & - & $52 / 64,254(0.08)$ & $\begin{array}{l}154 / 259,550 \\
(0.059)\end{array}$ & $\begin{array}{c}\text { VUS } \\
(\mathrm{BrS}+\mathrm{stnQT})\end{array}$ & $\mathrm{P}$ & VUS \\
\hline & p.(Asn547Ser) & rs768614762 & - & $1 / 53,304(0.001)$ & - & $\mathrm{BrS}+\operatorname{stn} \mathrm{QT}$ & VUS & VUS \\
\hline & p.(Arg632Arg) & - & - & - & - & $\mathrm{BrS}+\operatorname{stnQT}$ & - & VUS \\
\hline & p.(Glu1115Lys) & rs199473391 & - & - & - & $\mathrm{BrS}+\operatorname{stn} \mathrm{QT}$ & VUS & VUS \\
\hline & p.(Arg1780His) & rs756829999 & - & - & $\begin{array}{c}3 / 239,226 \\
(0.0012)\end{array}$ & $\mathrm{BrS}+\operatorname{stnQT}$ & VUS & VUS \\
\hline & p.(Glu1829_Gln1833dup) & - & - & - & - & $\mathrm{BrS}+\operatorname{stnQT}$ & - & VUS \\
\hline & p.(Arg1880Gln) & rs182208896 & - & $\begin{array}{c}59 / 120,512 \\
(0.048)\end{array}$ & $\begin{array}{c}166 / 276,890 \\
(0.059)\end{array}$ & $\begin{array}{c}\text { VUS } \\
(\mathrm{BrS}+\operatorname{stn} Q \mathrm{~T})\end{array}$ & VUS & VUS \\
\hline & p.(Val2014Ile) & rs199473660 & - & - & $\begin{array}{c}91 / 268,760 \\
(0.033)\end{array}$ & $\begin{array}{c}\text { VUS } \\
(\mathrm{BrS}+\operatorname{stn} Q \mathrm{~T})\end{array}$ & VUS & VUS \\
\hline & p.(Asp2130Asn) & rs199473392 & - & - & $\begin{array}{c}29 / 237,712 \\
(0,012)\end{array}$ & $\mathrm{BrS}+\operatorname{stnQT}$ & VUS & VUS \\
\hline CACNA2D1 & p.(Ser755Thr) & rs151327713 & $0.1047 / 0.0 / 0.0692$ & $93 / 120,134(0.07)$ & $\begin{array}{c}233 / 275,628 \\
(0.084)\end{array}$ & CM111612 & VUS & B \\
\hline CACNB2 & p.(Ser481Leu) & - & - & - & - & $\mathrm{BrS}+\operatorname{stn} \mathrm{QT}$ & - & VUS \\
\hline \multirow{9}{*}{ KCNH2 } & p.(Glu50Asp) & rs199472841 & - & - & - & CM094307 & VUS & LP \\
\hline & p.(Arg164Cys) & - & - & - & $\begin{array}{l}1 / 30,828 \\
(0.0032)\end{array}$ & CM141125 & - & VUS \\
\hline & p.(Ile560Thr) & - & - & - & - & CM156034 & - & $\mathrm{P}$ \\
\hline & $\begin{array}{l}\text { p.(Asn588Lys) } \\
\text { c. (1764C>A) }\end{array}$ & rs104894021 & - & - & - & CM040083 & $\mathrm{P}$ & $\mathrm{P}$ \\
\hline & $\begin{array}{l}\text { p.(Asn588Lys) } \\
\text { c. (1764C>G) }\end{array}$ & rs104894021 & - & - & - & CM040082 & $\mathrm{P}$ & $\mathrm{P}$ \\
\hline & p.(Thr618Ile) & rs199472947 & - & - & - & CM111008 & VUS & $\mathrm{P}$ \\
\hline & p.(Ser631Ala) & - & - & - & - & - & - & LP \\
\hline & p.(Trp927Gly) & - & - & - & - & CM141126 & - & LP \\
\hline & p.(Arg1135His) & rs199473547 & - & - & $\begin{array}{l}2 / 30,890 \\
(0.0064) \\
\end{array}$ & CM086664 & VUS & VUS \\
\hline \multirow{4}{*}{ KCNJ2 } & p.(Asp172Asn) & rs104894584 & - & - & - & CM051549 & $\mathrm{P}$ & $\mathrm{P}$ \\
\hline & p.(Glu299Val) & rs786205817 & - & - & - & CM131839 & LP & $\mathrm{P}$ \\
\hline & p.(Met301Lys) & - & - & - & - & CM1110289 & - & $\mathrm{P}$ \\
\hline & p.(Lys346Thr) & - & - & - & - & Epilepsy + stnQT & - & VUS \\
\hline
\end{tabular}


Table 1. Cont.

\begin{tabular}{|c|c|c|c|c|c|c|c|c|}
\hline Gene & Protein & dbSNP & $\begin{array}{c}\text { EVS MAF (\%) } \\
\text { EA/AA/All }\end{array}$ & ExAC Alleles (\%) & gnomAD Alleles (\%) & HGMD (DM) & ClinVar & $\begin{array}{l}\text { ACMG/AMP } \\
\text { Classification }\end{array}$ \\
\hline \multirow{5}{*}{ KCNQ1 } & p.(Phe279Ile) & - & - & - & - & CM157723 & - & LP \\
\hline & p.(Val307Leu) & rs120074195 & - & - & - & CM41383 & $\mathrm{P}$ & $\mathrm{P}$ \\
\hline & p.(Val141Met) & - & - & - & - & $\mathrm{SQTS}+\mathrm{AF}$ & - & P \\
\hline & p.(Ile274Val) & rs199472728 & $0.0116 / 0.0 / 0.0077$ & $39 / 119,484(0.03)$ & $\begin{array}{c}49 / 276,714 \\
(0.0177)\end{array}$ & SIDS & $\begin{array}{c}\text { LP } \\
\text { (LQTS, SIDS) }\end{array}$ & VUS \\
\hline & p.(Arg259His) & rs199472720 & - & 1/104,552 (0.0009) & $\begin{array}{c}5 / 271,834 \\
(0.0018) \\
\end{array}$ & LQTS & LP/P (LQTS) & VUS \\
\hline SCN5A & p.(Arg689His) & rs199473145 & 0.0118/0.0/0.0078 & $14 / 120,204(0.011)$ & $\begin{array}{c}25 / 245,708 \\
(0.01)\end{array}$ & $\begin{array}{c}\text { VUS } \\
(\mathrm{BrS}+\operatorname{stnQT})\end{array}$ & VUS & VUS \\
\hline$S L C 4 A 3$ & p.(Arg370His) & - & - & - & - & CM1717443 & - & LP \\
\hline
\end{tabular}

Pathogenic and additional variants associated with Short QT Syndrome. DM: Disease Mutation, EVS: Exome Variant Server (EA: European-American, AA: African-American, All: All

populations), ExAC: Exome Aggregation Consortium, gnomAD: Genome Aggregation Database, HGMD: Human Genome Mutation Database, MAF: Minor Allele Frequency, ACMG/AMP: American College of Medical Genetics and Genomics/Association for Molecular Pathology, ClinVar: Clinical Variation, P: Pathogenic, LP: Likely Pathogenic, VUS: Variant of Uncertain Significance, B: Benign, LQTS: Long QT Syndrome, SIDS: Sudden Infant Death Syndrome, BrS: Brugada Syndrome, AF: Atrial Fibrillation, stnQT: shorter than normal QT. 
Table 2. Classification in silico of variants.

\begin{tabular}{|c|c|c|c|c|c|c|c|c|c|c|c|c|}
\hline Gene & Protein & CADD & MetaSVM & GERP & M-CAP & MKL & MetaLR & MT & MA & PhyloP & PROVEAN & PPH2 \\
\hline \multirow{10}{*}{ CACNA1C } & p.(Ala39Val) & $\mathrm{N}$ & $\mathrm{N}$ & NC & PD & $\mathrm{D}$ & $\mathrm{N}$ & DC & $\mathrm{N}$ & NC & $\mathrm{N}$ & PD \\
\hline & p.(Gly490Arg) & $\mathrm{N}$ & $\mathrm{N}$ & $\mathrm{NC}$ & $\mathrm{N}$ & $\mathrm{N}$ & $\mathrm{D}$ & $\mathrm{DC}$ & $\mathrm{N}$ & $\mathrm{NC}$ & $\mathrm{N}$ & $\mathrm{N}$ \\
\hline & p.(Asn547Ser) & $\mathrm{N}$ & $\mathrm{D}$ & NC & PD & $\mathrm{N}$ & $\mathrm{D}$ & DC & $\mathrm{N}$ & NC & $\mathrm{N}$ & B \\
\hline & p.(Arg632Arg) & $\mathrm{N}$ & $\mathrm{D}$ & $\mathrm{NC}$ & $\mathrm{D}$ & $\mathrm{D}$ & $\mathrm{D}$ & DC & $\mathrm{N}$ & NC & $\mathrm{N}$ & $\mathrm{N}$ \\
\hline & p.(Glu1115Lys) & $\mathrm{N}$ & $\mathrm{D}$ & $\mathrm{NC}$ & $\mathrm{N}$ & $\mathrm{D}$ & $\mathrm{D}$ & DC & $\mathrm{N}$ & NC & $\mathrm{N}$ & PD \\
\hline & p.(Arg1780His) & $\mathrm{N}$ & $\mathrm{D}$ & NC & $\mathrm{PD}$ & $\mathrm{N}$ & $\mathrm{D}$ & DC & $\mathrm{N}$ & NC & $\mathrm{N}$ & $\mathrm{N}$ \\
\hline & p.(Glu1829_Gln1833dup) & PD & $\mathrm{D}$ & $\mathrm{C}$ & $\mathrm{D}$ & $\mathrm{D}$ & $\mathrm{D}$ & DC & $\mathrm{N}$ & $\mathrm{C}$ & PD & PD \\
\hline & p.(Arg1880Gln) & $\mathrm{N}$ & $\mathrm{D}$ & $\mathrm{NC}$ & $\mathrm{N}$ & $\mathrm{D}$ & $\mathrm{D}$ & DC & $\mathrm{N}$ & $\mathrm{NC}$ & $\mathrm{N}$ & $\mathrm{N}$ \\
\hline & p.(Val2014lle) & $\mathrm{N}$ & $\mathrm{D}$ & $\mathrm{NC}$ & $\mathrm{N}$ & $\mathrm{D}$ & $\mathrm{D}$ & $\mathrm{DC}$ & $\mathrm{N}$ & $\mathrm{NC}$ & $\mathrm{N}$ & B \\
\hline & p.(Asp2130Asn) & $\mathrm{N}$ & $\mathrm{N}$ & $\mathrm{NC}$ & $\mathrm{N}$ & $\mathrm{D}$ & $\mathrm{N}$ & DC & $\mathrm{N}$ & $\mathrm{NC}$ & $\mathrm{N}$ & PD \\
\hline CACNA2D1 & p.(Ser755Thr) & $\mathrm{N}$ & $\mathrm{N}$ & $\mathrm{NC}$ & $\mathrm{PD}$ & $\mathrm{D}$ & $\mathrm{N}$ & $\mathrm{DC}$ & $\mathrm{N}$ & $\mathrm{NC}$ & $\mathrm{PD}$ & $\mathrm{N}$ \\
\hline CACNB2 & p.(Ser481Leu) & $\mathrm{N}$ & $\mathrm{D}$ & $\mathrm{NC}$ & $\mathrm{N}$ & $\mathrm{D}$ & $\mathrm{D}$ & DC & $\mathrm{N}$ & $\mathrm{NC}$ & $\mathrm{N}$ & $\mathrm{N}$ \\
\hline \multirow{9}{*}{ KCNH2 } & p.(Glu50Asp) & PD & $\mathrm{D}$ & $\mathrm{C}$ & $\mathrm{D}$ & $\mathrm{D}$ & $\mathrm{D}$ & DC & $\mathrm{N}$ & $\mathrm{C}$ & $\mathrm{PD}$ & PD \\
\hline & p.(Arg164Cys) & $\mathrm{N}$ & $\mathrm{D}$ & $\mathrm{NC}$ & $\mathrm{D}$ & $\mathrm{D}$ & $\mathrm{D}$ & DC & $\mathrm{N}$ & $\mathrm{NC}$ & $\mathrm{N}$ & $\mathrm{N}$ \\
\hline & p.(Ile560Thr) & $\mathrm{PD}$ & $\mathrm{D}$ & $\mathrm{NC}$ & $\mathrm{D}$ & $\mathrm{D}$ & $\mathrm{D}$ & DC & PD & $\mathrm{NC}$ & $\mathrm{PD}$ & PD \\
\hline & $\begin{array}{l}\text { p.(Asn588Lys) } \\
\text { c.(1764C >A) }\end{array}$ & PD & $\mathrm{D}$ & C & $\mathrm{D}$ & $\mathrm{D}$ & $\mathrm{D}$ & DC & $\mathrm{N}$ & C & $\mathrm{N}$ & $\mathrm{PD}$ \\
\hline & p.(Asn588Lys)c. $(1764 \mathrm{C}>\mathrm{G})$ & $\mathrm{PD}$ & $\mathrm{D}$ & C & $\mathrm{D}$ & $\mathrm{D}$ & $\mathrm{D}$ & DC & $\mathrm{N}$ & C & $\mathrm{N}$ & PD \\
\hline & p.(Thr618Ile) & $\mathrm{N}$ & $\mathrm{D}$ & $\mathrm{C}$ & $\mathrm{D}$ & $\mathrm{D}$ & $\mathrm{D}$ & DC & PD & $\mathrm{C}$ & $\mathrm{N}$ & PD \\
\hline & p.(Ser631Ala) & $\mathrm{PD}$ & $\mathrm{D}$ & $\mathrm{C}$ & $\mathrm{D}$ & $\mathrm{D}$ & $\mathrm{D}$ & $\mathrm{DC}$ & $\mathrm{N}$ & $\mathrm{C}$ & $\mathrm{N}$ & PD \\
\hline & p.(Trp927Gly) & PD & $\mathrm{D}$ & $\mathrm{NC}$ & $\mathrm{D}$ & $\mathrm{D}$ & $\mathrm{D}$ & DC & $\mathrm{N}$ & NC & $\mathrm{N}$ & B \\
\hline & p.(Arg1135His) & $\mathrm{N}$ & $\mathrm{D}$ & $\mathrm{NC}$ & $\mathrm{D}$ & $\mathrm{D}$ & $\mathrm{D}$ & DC & $\mathrm{N}$ & $\mathrm{NC}$ & $\mathrm{N}$ & B \\
\hline \multirow{4}{*}{ KCNJ2 } & p.(Asp172Asn) & PD & $\mathrm{D}$ & $\mathrm{NC}$ & $\mathrm{D}$ & $\mathrm{D}$ & $\mathrm{D}$ & DC & $\mathrm{N}$ & $\mathrm{NC}$ & PD & PD \\
\hline & p.(Glu299Val) & $\mathrm{N}$ & $\mathrm{D}$ & $\mathrm{C}$ & $\mathrm{D}$ & $\mathrm{D}$ & $\mathrm{D}$ & DC & $\mathrm{N}$ & $\mathrm{C}$ & PD & PD \\
\hline & p.(Met301Lys) & PD & $\mathrm{D}$ & $\mathrm{C}$ & $\mathrm{D}$ & $\mathrm{D}$ & $\mathrm{D}$ & DC & $\mathrm{N}$ & $\mathrm{C}$ & PD & PD \\
\hline & p.(Lys346Thr) & $\mathrm{N}$ & $\mathrm{D}$ & C & $\mathrm{D}$ & $\mathrm{D}$ & $\mathrm{D}$ & DC & $\mathrm{N}$ & C & PD & PD \\
\hline \multirow{5}{*}{ KCNQ1 } & p.(Phe279Ile) & PD & $\mathrm{D}$ & $\mathrm{C}$ & $\mathrm{D}$ & PD & $\mathrm{D}$ & DC & $\mathrm{N}$ & $\mathrm{C}$ & $\mathrm{D}$ & PD \\
\hline & p.(Val307Leu) & $\mathrm{N}$ & $\mathrm{D}$ & $\mathrm{C}$ & $\mathrm{D}$ & $\mathrm{D}$ & $\mathrm{D}$ & DC & PD & $\mathrm{C}$ & $\mathrm{D}$ & PD \\
\hline & p.(Val141Met) & PD & $\mathrm{D}$ & $\mathrm{C}$ & $\mathrm{D}$ & $\mathrm{D}$ & $\mathrm{D}$ & DC & $\mathrm{N}$ & C & $\mathrm{N}$ & PD \\
\hline & p.(Ile274Val) & $\mathrm{N}$ & $\mathrm{N}$ & $\mathrm{NC}$ & PD & $\mathrm{D}$ & $\mathrm{N}$ & DC & $\mathrm{N}$ & $\mathrm{NC}$ & PD & $\mathrm{N}$ \\
\hline & p.(Arg259His) & $\mathrm{N}$ & $\mathrm{D}$ & $\mathrm{NC}$ & $\mathrm{D}$ & $\mathrm{D}$ & $\mathrm{PD}$ & $\mathrm{DC}$ & $\mathrm{N}$ & $\mathrm{NC}$ & $\mathrm{N}$ & $\mathrm{B}$ \\
\hline SCN5A & p.(Arg689His) & $\mathrm{N}$ & $\mathrm{D}$ & $\mathrm{NC}$ & $\mathrm{PD}$ & $\mathrm{D}$ & $\mathrm{PD}$ & DC & $\mathrm{N}$ & $\mathrm{NC}$ & $\mathrm{N}$ & $\mathrm{PD}$ \\
\hline$S L C 4 A 3$ & p.(Arg370His) & $\mathrm{N}$ & $\mathrm{D}$ & C & $\mathrm{D}$ & $\mathrm{D}$ & $\mathrm{D}$ & DC & $\mathrm{N}$ & C & $\mathrm{D}$ & PD \\
\hline
\end{tabular}

Classification in silico of variants. Combined Annotation Dependent Depletion -CADD-, Genomic Evolutionary Rate Profiling -GERP-, MetaSVM, Mendelian Clinically Applicable Pathogenicity Score -M-CAP-, Fathmm-MKL, MetaLR, Mutation Taster -MT-, Mutation Assessor -MA-, Phylogenetic Analysis -PhyloP-, Protein Variation Effect Analyzer -PROVEAN-, Polymorphism Phenotyping v2 -PolyPhen2, PPH2-, DC: Disease Causing, B: Benign, D: Deleterious/Damaging, PD: Probably Damaging/Deleterious, N: Neutral, C: Conserved aminoacid, NC: No conserved aminoacid. 
Table 3. Classification of variants following American College of Medical Genetics and Genomics/Association for Molecular Pathology (ACMG/AMP) criteria.

\begin{tabular}{|c|c|c|c|c|c|c|c|c|c|c|}
\hline Gene & Protein & $\begin{array}{c}\text { Population } \\
\text { Data }\end{array}$ & $\begin{array}{c}\text { Computational } \\
\text { and Predictive } \\
\text { Data }\end{array}$ & $\begin{array}{c}\text { Functional } \\
\text { Data }\end{array}$ & $\begin{array}{c}\text { Segregation } \\
\text { Data }\end{array}$ & $\begin{array}{l}\text { De novo } \\
\text { Data }\end{array}$ & Allelic Data & $\begin{array}{c}\text { Other } \\
\text { Database }\end{array}$ & Other Data & $\begin{array}{l}\text { ACMG/AMP } \\
\text { Classification }\end{array}$ \\
\hline \multirow{10}{*}{ CACNA1C } & p.(Ala39Val) & PM2 & C & PP2 & NA & PM6 & NA & C & NA & VUS \\
\hline & p.(Gly490Arg) & BS1 & C & PP2 & NA & NA & NA & BP6 & NA & VUS \\
\hline & p.(Asn547Ser) & PM2 & $\mathrm{C}$ & PP2 & NA & PM6 & NA & $\mathrm{C}$ & NA & VUS \\
\hline & p.(Arg632Arg) & PM2 & C & PP2 & NA & PM6 & NA & $\mathrm{C}$ & NA & VUS \\
\hline & p.(Glu1115Lys) & PM2 & $\mathrm{C}$ & PP2 & NA & NA & NA & $\mathrm{C}$ & NA & VUS \\
\hline & p.(Arg1780His) & PM2 & $\mathrm{C}$ & PP2 & NA & PM6 & NA & C & NA & VUS \\
\hline & p.(Glu1829_Gln1833dup) & PM2 & PP3 & PP2 & NA & NA & NA & C & NA & VUS \\
\hline & p. (Arg1880Gln) & PM2 & $\mathrm{C}$ & PP2 & NA & PM6 & NA & $\mathrm{C}$ & NA & VUS \\
\hline & p.(Val2014Ile) & PM2 & C & PP2 & NA & PM6 & NA & C & NA & VUS \\
\hline & p.(Asp2130Asn & PM2 & C & PP2 & NA & NA & NA & $\mathrm{C}$ & NA & VUS \\
\hline CACNA2D1 & p.(Ser755Thr) & BS1 & $\mathrm{C}$ & BS3 & NA & NA & NA & $\mathrm{C}$ & NA & $\mathrm{B}$ \\
\hline CACNB2 & p.(Ser481Leu) & PM2 & $\mathrm{C}$ & PS3 & $\mathrm{NA}$ & NA & NA & $\mathrm{C}$ & $\mathrm{NA}$ & VUS \\
\hline \multirow{9}{*}{ KCNH2 } & p.(Glu50Asp) & PM2 & PP3 & PP2 & NA & PM6 & NA & $\mathrm{C}$ & NA & LP \\
\hline & p.(Arg164Cys) & PM2 & C & PP2 & NA & NA & NA & C & NA & VUS \\
\hline & p.(Ile560Thr) & PM2 & PP3 & PS3 & NA & PM6 & NA & $\mathrm{C}$ & PP4 & $\mathrm{P}$ \\
\hline & $\begin{array}{l}\text { p.(Asn588Lys) } \\
\text { c. }(1764 \mathrm{C}>\mathrm{A})\end{array}$ & PM2 & PP3 & PS3 & NA & PM6 & NA & PP5 & PP4 & $\mathrm{P}$ \\
\hline & $\begin{array}{l}\text { p.Asn588Lys } \\
\text { c. }(1764 C>G)\end{array}$ & PM2 & PP3 & PS3 & NA & PM6 & NA & PP5 & PP4 & $\mathrm{P}$ \\
\hline & p.(Thr618Ile) & PM2 & PP3 & PS3 & NA & PM6 & NA & C & PP4 & $\mathrm{P}$ \\
\hline & p.(Ser631Ala) & PM2 & PP3 & PS3 & NA & PM6 & NA & $\mathrm{C}$ & NA & LP \\
\hline & p.(Trp927Gly) & PM2 & РP3 & PP2 & NA & PM6 & NA & $\mathrm{C}$ & NA & LP \\
\hline & p.(Arg1135His) & PM2 & $\mathrm{C}$ & PS3 & PP1 & NA & NA & $\mathrm{C}$ & NA & VUS \\
\hline \multirow{4}{*}{ KCNJ2 } & p.(Asp172Asn) & PM2 & PP3 & PS3 & NA & PM6 & NA & PP5 & PP4 & $\mathrm{P}$ \\
\hline & p.(Glu299Val) & PM2 & $\mathrm{C}$ & PS3 & NA & PM6 & NA & $\mathrm{C}$ & PP4 & $\mathrm{P}$ \\
\hline & p.(Met301Lys) & PM2 & PP3 & PS3 & NA & PM6 & NA & $\mathrm{C}$ & C & $\mathrm{P}$ \\
\hline & p.(Lys346Thr) & PM2 & $\mathrm{C}$ & PP2 & $\mathrm{NA}$ & PM6 & NA & $\mathrm{C}$ & $\mathrm{C}$ & VUS \\
\hline \multirow{5}{*}{ KCNQ1 } & p.(Phe279Ile) & PM2 & PP3 & PP2 & NA & PM6 & NA & $\mathrm{C}$ & NA & LP \\
\hline & p.(Val307Leu) & PM2 & PP3 & PS3 & NA & PM6 & NA & PP5 & NA & $\mathrm{P}$ \\
\hline & p.(Val141Met) & PM2 & PP3 & PS3 & PP1 & PS2 & NA & PP5 & PP4 & $\mathrm{P}$ \\
\hline & p.(Ile274Val) & BS1 & $\mathrm{C}$ & PP2 & NA & NA & NA & $\mathrm{C}$ & NA & VUS \\
\hline & p.(Arg259His) & PM2 & $\mathrm{C}$ & PS3 & NA & NA & NA & $\mathrm{C}$ & NA & VUS \\
\hline SCN5A & p.(Arg689His) & PM2 & $\mathrm{C}$ & PS3 & $\mathrm{NA}$ & PM6 & NA & $C$ & $\mathrm{C}$ & VUS \\
\hline SLC4A3 & p.(Arg370His) & PM2 & PP3 & PS3 & NA & PM6 & NA & C & PP4 & LP \\
\hline
\end{tabular}

Classification of variants following American College of Medical Genetics and Genomics/Association for Molecular Pathology (ACMG/AMP) criteria. B: Benign, BA: Evidence of benign impact Stand-alone, BP: Evidence of benign impact Supporting, BS: Evidence of benign impact Strong, C: Contradictory data, LP: Likely Pathogenic, NA: Not available data, P: Pathogenic, PM: Evidence of Pathogenicity Moderate, PP: Evidence of Pathogenicity Supporting, PS: Evidence of Pathogenicity Strong, PVS: Evidence of Pathogenicity Very Strong, VUS: Variant of Uncertain Significance. 


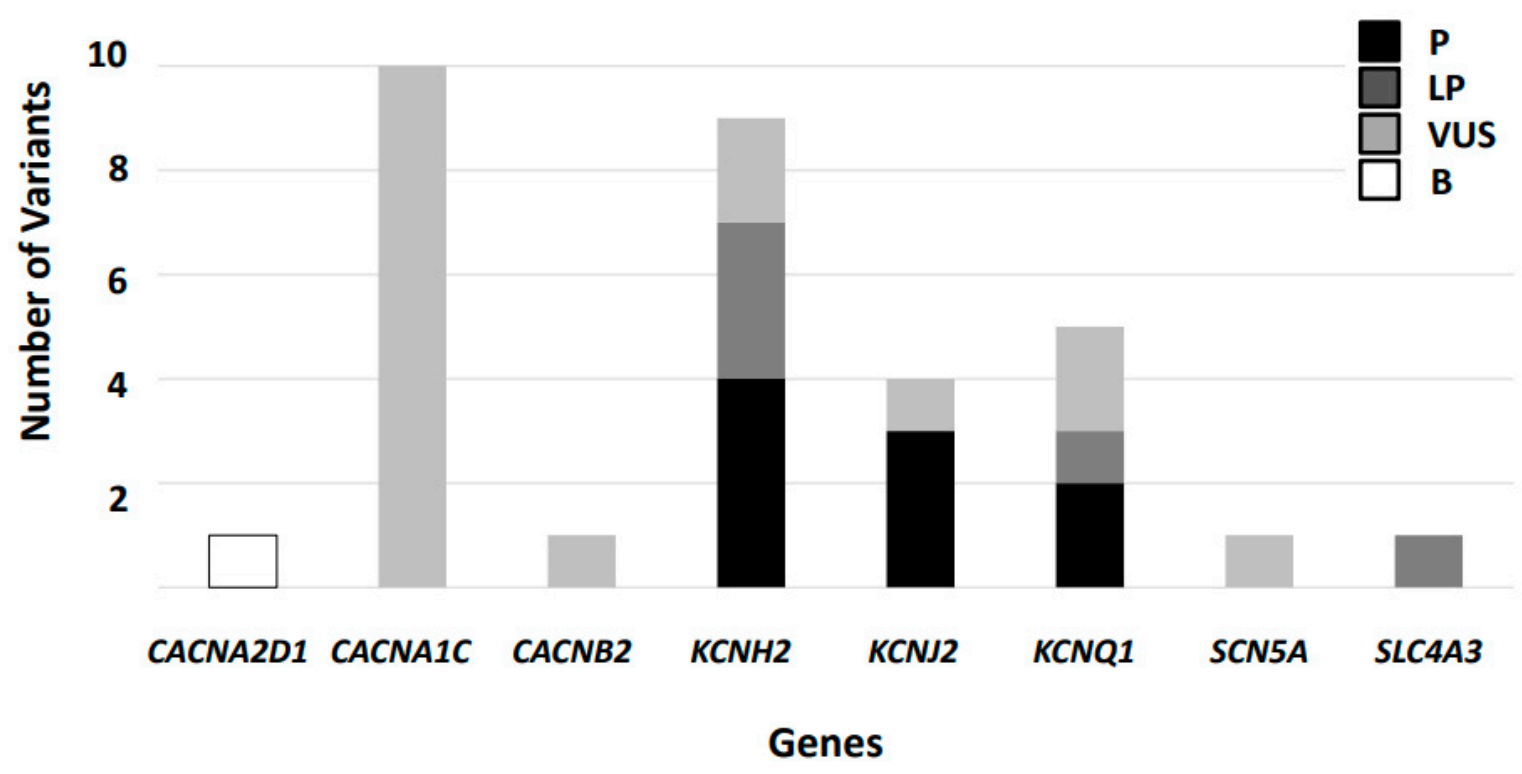

Figure 1. Classification of variants reported in Short QT Syndrome following American College of Medical Genetics and Genomics/Association for Molecular Pathology guidelines. Thirty-two variants were identified but only nine remains as definitively deleterious. P: Pathogenic, LP: Likely Pathogenic, VUS: Variant of Uncertain of Significance, B: Benign.

\subsection{Other Variants Potentially Associated with Short QT Syndrome in Calcium Channels}

In 2007, three probands with a BrS phenotype and short QT intervals were published. Genetic analysis identified missense variants in CACNA1C: p.(Ala39Val) -CM070048-, p.(Gly490Arg) and CACNB2: p.(Ser481Leu). In vitro studies confirmed loss-of-function in calcium channel activity in p.(Ala39Val) [16] but Simms and Zamponi did not identify functional effects [17], although this last manuscript used the neuronal Cav1.2, not the cardiac isoform. Considering all data, including contradictory in silico predictions (Table 2), p.(Ala39Val) should be classified as VUS for SQTS following ACMG/AMP recommendations (Tables 1 and 3, Figure 1). The p.(Gly490Arg) variant (rs121912775, CM070047) in CACNA1C is identified in low frequencies. The variant is associated with LQTS and BrS, playing an ambiguous role. This contradictory effect is supported by differing in silico predictions (Table 2). The p.(Gly490Arg) variant should be classified as VUS for SQTS following ACMG/AMP recommendations (Tables 1 and 3, Figure 1).

Five rare variants in patients showing BrS and shorter than normal QT intervals were reported in the CACNA1C gene [18]. The first variant p.(Glu1115Lys) -rs199473391, CM109282- has not been identified in global databases so far. However, the same rare variant was recently identified in a patient showing an enlarged QT interval [19]. Considering all data, including contradictory in silico predictions (Table 2), p.(Glu1115Lys) should be classified as VUS for SQTS following ACMG/AMP recommendations (Tables 1 and 3, Figure 1). The variant p.(Glu1829_Gln1833dup) -CI109266- was identified in a phenotype of BrS with a shorter than normal QT interval. It has not been identified in global databases and in silico databases predict a deleterious effect (Table 2). Considering all data, p.(Glu1829_Gln1833dup) should be classified as VUS for SQTS following ACMG/AMP recommendations (Tables 1 and 3, Figure 1). The third variant was p.(Arg1880Gln) -rs182208896, CM109283-. It has not been identified in global databases but was identified in other reports highlighting the need for a cautious clinical interpretation. Due to conflicting information, including contradictory in silico prediction (Table 2), p.(Arg1880Gln) should be classified as VUS for SQTS following ACMG/AMP recommendations (Tables 1 and 3, Figure 1). The fourth variant was p.(Val2014Ile) (rs199473660, CM109284). In global databases has not been identified but it was identified in other reports highlighting a cautious interpretation, and in silico databases show a contradictory role (Table 2). Considering all divergent data, p.(Val2014Ile) should 
be classified as VUS for SQTS following ACMG/AMP recommendations (Tables 1 and 3, Figure 1). Finally, the fifth variant was p.(Asp2130Asn) (rs199473392, CM109285). Due to conflicting information, including contradictory in silico prediction (Table 2), p.(Asp2130Asn) should be classified as VUS for SQTS following ACMG/AMP recommendations (Tables 1 and 3, Figure 1).

In 2013, three more variants were identified in CACNA1C in patients showing BrS and shorter than normal QT intervals [20]. The first variant was p.(Asn547Ser) -rs768614762, CM133491-. In global populations, it has been identified at low frequency and in silico databases show a conflicting role (Table 2). Considering all incongruous data, p.(Asn547Ser) should be classified as VUS for SQTS following ACMG/AMP recommendations (Tables 1 and 3, Figure 1). The second variant was p.(Arg632Arg) -CS133492-. It produces no amino acid change, but the codon 1896 is the first nucleotide of exon 14 and causes a splicing error. Such a splicing error results in an exon skipping and a frame-shift, thereby a premature termination codon, which in turn causes the nonsense mutation-mediated decay of mRNA (NMD). However, the corrected QT interval was within the normal range (383 ms). In 2014, the same group reported that mutant mRNA with a c. $(1896 \mathrm{G}>\mathrm{A})$ substitution may be diminished by nonsense-mediated mRNA decay [21]. Due to conflicting information, including in silico prediction (Table 2), p.(Arg632Arg) should be classified as VUS for SQTS (Tables 1 and 3, Figure 1). The third variant was p.(Arg1780His) (rs756829999, CM133493). Due to conflicting information reported so far, p.(Arg1780His) should be classified as VUS for SQTS following ACMG/AMP recommendations (Tables 1 and 3, Figure 1).

Finally, the CACNB2_p.(Ser481Leu) variant (CM070049) was reported in a family showing BrS phenotype and short QT interval [16]. In vitro studies confirmed loss-of-function in calcium channel activity but in silico databases show a contradictory role (Table 2). Considering all divergence data, p.(Ser481Leu) should be classified as VUS for SQTS following current ACMG/AMP recommendations (Tables 1 and 3, Figure 1).

\subsection{The KCNH2 Gene}

In 2003, three families with several members diagnosed with SQTS were reported [22]. The p.(Asn588Lys) variant (rs104894021, CM040082/CM040083) was identified in KCNH2. Conclusive data concerning pathogenicity was recently reported [23], even using human-induced pluripotent stem cell-derived cardiomyocytes (hiPSC-CMs) [24,25] supporting a deleterious role previously proposed in 2003. Considering all data, including in silico analysis (Table 2), p.(Asn588Lys) should be classified as Pathogenic for SQTS following ACMG/AMP recommendations (Tables 1 and 3, Figure 1).

In 2008, a novel variant in KCNH2, p.(Arg1135His) -rs199473547, CM086664- was reported [26]. Functional studies showed a potential repolarization effect but no additional studies have been reported. Considering all data, including contradictory in silico prediction (Table 2), p.(Arg1135His) should be classified as VUS for SQTS following ACMG/AMP recommendations (Tables 1 and 3, Figure 1).

One additional rare variant, p.(Glu50Asp) -rs199472841, CM094307-, was reported [27]. It was previously classified as pathogenic despite no familial segregation, based on in silico predictions (Table 2). Considering all data, p.(Glu50Asp) should be classified as Likely Pathogenic for SQTS following ACMG/AMP recommendations (Tables 1 and 3, Figure 1) due to lack of conclusive studies concerning pathogenicity in SQTS. In 2011, several relatives showing SQTS were reported [28]. Genetic analysis identified the p.(Thr618Ile) variant (rs199472947, CM111008) in KCNH2. Functional analysis identified a mechanism for its potential pathogenic role [29]. Recently, Guo et al. published a study using human-induced pluripotent stem cell-derived cardiomyocytes (hiPSC-CMs), facilitating our understanding of the mechanism of the disease and confirming its deleterious role in SQTS [30].Considering all published data, including in silico prediction (Table 2), p.(Thr618Ile) should be classified as Pathogenic for SQTS following ACMG/AMP recommendations (Tables 1 and 3 , Figure 1).

In 2014, an additional rare variant -p.(Arg164Cys), CM141125- was reported in a cohort of BrS patients [31]. No additional studies have been published to date. Considering all data, including 
incongruous in silico prediction (Table 2), p.(Arg164Cys) should be classified as VUS for SQTS following ACMG/AMP recommendations (Tables 1 and 3, Figure 1). The same report of Wang et al. also identified the p.(Trp927Gly) variant (CM141126) in $\mathrm{KCNH} 2$ as associated with SQTS Despite one publication reporting its pathogenicity, no additional studies support this role. Considering all published data, including in silico prediction (Table 2), p.(Trp927Gly) should be classified as Likely Pathogenic for SQTS (Tables 1 and 3, Figure 1).

In 2015, another variant in KCNH2 p.(Ile560Thr) -rs372725107, CM156034- was identified in a SQTS patients as pathogenic. This role is supported by a recent publication [32]. Considering all data, p.(Ile560Thr) should be classified as Pathogenic for SQTS following ACMG/AMP recommendations, despite no familial phenotype-genotype segregation (Tables 1 and 3, Figure 1).

Recently, a novel variant in $\mathrm{KCNH} 2$, p.(Ser631Ala), was identified in a family with several relatives who died suddenly at young ages [33]. None of those who died were diagnosed with SQTS but three living relatives were diagnosed and carried the same novel genetic variant -KCNH2_p.(Ser631Ala)-. In 2018, a functional study was published concerning this rare variant showed deleterious in vitro role [34]. Considering all data, including in silico prediction (Table 2), p.(Ser631Ala) should be classified as Likely Pathogenic for SQTS (Tables 1 and 3, Figure 1).

\subsection{The KCNJ2 Gene}

The first reported variant associated with SQTS in KCNJ2 was p.(Asp172Asn) -rs104894584, CM051549-. Familial genetic analysis identified the variant in two clinically affected relatives [35]. Functional analysis provided evidence for pro-arrhythmic effects in perpetuating and facilitating re-entrant excitation waves. Considering all published data, including in silico predictions (Table 2), p.(Asp172Asn) should be classified as Pathogenic (Tables 1 and 3, Figure 1).

In 2012, the p.(Met301Lys) variant-CM1110289- was reported [36]. A second publication identified p.(Met301Lys) in a young patient with juvenile-onset atrial fibrillation who also carried the rare genetic variant p.(Gly299Asp) in KCNQ1, which was considered the cause of disease [37]. Considering all data, including in silico predictions (Table 2), p.(Met301Lys) should be classified as Pathogenic for SQTS following ACMG/AMP recommendations, despite no familial segregation (Tables 1 and 3, Figure 1).

The third variant, p.(Glu299Val) -rs786205817, CM131839-, was reported in 2013 [38]. Considering all data, including in silico predictions (Table 2), p.(Glu299Val) should be classified as Pathogenic for SQTS (Tables 1 and 3, Figure 1).

\subsection{Other Variants in KCNJ2 Potentially Associated with Short QT Syndrome}

In 2014, monozygotic twins displaying short QT intervals and autism-epilepsy syndrome were reported [39]. Genetic analysis identified a novel variant in KCNJ2, p.(Lys346Thr) -CM148325-. Considering all data, p.(Lys346Thr) should be classified as VUS for SQTS following ACMG/AMP recommendations (Tables 1 and 3, Figure 1).

\subsection{The KCNQ1 Gene}

Two variants associated with SQTS and classified as DM were identified in KCNQ1: p.(Val307Leu) -rs120074195, CM041383- [40], and p.(Phe279Ile) -CM157723- [41]. Functional and in silico simulation of p.(Val307Leu) supported a pathogenic role (Table 2). Considering all data p.(Val307Leu) should be classified as Pathogenic for SQTS following ACMG/AMP recommendations, despite lack of familial segregation (Tables 1 and 3, Figure 1). For p.(Phe279Ile), all data support a Likely Pathogenic role for SQTS, despite the lack of familial segregation (Tables 1 and 3, Figure 1).

\subsection{Other Variants in KCNQ1 Potentially Associated with Short QT Syndrome}

In 2005, a novel in utero diagnosis characterized by atrial fibrillation and SQTS was reported. Genetic analysis identified a de novo variant in KCNQ1, p.(Val141Met) -CM056972- [42]. Functional studies showed an alteration in function [43]. Posterior studies confirmed the curious phenotype in 
patients carrying the same variant [44,45]. Considering all data, including in silico predictions (Table 2), p.(Val141Met) should be classified as Pathogenic for SQTS following ACMG/AMP recommendations (Tables 1 and 3, Figure 1).

Another variant in KCNQ1, p.(Ile274Val) -rs199472728, CM070181-, was identified in a sudden infant death syndrome (SIDS) case [46]. This variant was identified with a frequency $>0.3 \%$, and in silico databases show a contradictory role (Table 2). Considering all data, p.(Ile274Val) should be classified as VUS for SQTS (Tables 1 and 3, Figure 1).

In 2006, the KCNQ1_p.(Arg259His) variant (rs199472720, CM064074) was reported in a LQTS patient [47]. An additional report confirmed its deleterious effect in vitro [48], and in silico databases show a contradictory role (Table 2). Considering all data, p.(Arg259His) should be classified as VUS for SQTS (Tables 1 and 3, Figure 1).

\subsection{The SCN5A Gene}

In 2004, Ackerman et al. analyzed apparently healthy individuals and identified the variant p.(Arg689His) -rs199473145, CM057209- [49]. One year later, the same variant was identified in a patient diagnosed with LQTS [50]. Further, Nakajima et al. identified the variant in a cohort of patients diagnosed with BrS [51]. In 2012, a publication identified the same rare variant in an asymptomatic 40-year-old male with family history of SD who had a Brugada-like ECG with short QT intervals [52]. Other reports have identified the same rare variant in global populations. Due to conflicting information, including frequencies and in silico databases (Table 2), p.(Arg689His) should be classified as VUS for SQTS following ACMG/AMP recommendations (Tables 1 and 3, Figure 1).

\subsection{The SLC4A3 Gene}

In 2017, a novel variant p.(Arg370His) in SLC4A3 was reported as associated with SQTS [53]. This variant (CM1717443) was identified in several clinically affected relatives of two families. Considering all published data (Table 2), p.(Arg370His) should be classified as Likely Pathogenic for SQTS (Tables 1 and 3, Figure 1).

\section{Discussion}

Pathogenic role of rare variants has been debated in almost all diseases areas but especially controversial in SQTS given the immediate impact of the lethality and reduced penetrance of many variants even in well established genes. Nowadays, 32 rare variants have been reported with a potential causative role in SQTS. These variants are reported in HGMD, ClinVar and PubMed. First two databases only archives submitted information available about a variant or condition from other public resources. In addition, both HGMD and ClinVar databases neither curates content and modifies interpretations independent of an explicit submission. Therefore, some of variants currently interpreted as DM may lead to confusion in clinical translation. One of parameters used in the published reports is in silico prediction; it is important to note that these algorithms are only mathematical approaches and are recognized to be poor predictors of diseases causation. An in silico prediction cannot faithfully reflect a human mechanistic pathway. Therefore, can be a marked divergence between in silico predictions and functional data, as we previously reported, and the use of bioinformatic tools can produce erroneous conclusions regarding pathogenicity [54]. Hence, despite included in ACMG/AMP recommendations, they represent the lowest level of evidence for pathogenicity classification. In consequence, a comprehensive analysis of all data published may help to clarify the real role of rare variants currently reported as potentially deleterious in SQTS, and, in addition, establish the main genes associated with conclusive clinical diagnosis of SQTS. In recent years, use of hiPSC-CMs help to solve this ambiguous pathogenic role, at least in large part of analyzed rare variants [24]. Therefore, hiPSC-CMs are able to recapitulate the single-cell phenotypic features of SQTS and provide novel opportunities to further elucidate the disease mechanism and test drug effects [25]. 
Current guidelines published in 2015 recommend analysis of five genes (KCNH2, KCNQ1, KCNJ2, $C A C N A 1 C$, and $C A C N B 2 b$ ) in suspected SQTS cases [4]. Concerning calcium genes, all variants reported so far are associated with patients showing $\mathrm{BrS}$ and shorter QT intervals, but not a conclusive clinical diagnosis of SQTS. This is an important point in the final clinical classification because of a variant could be pathogenic for a phenotype showing a reduction of the QT interval but not SQTS as entity. The CACNA2D1 gene was also reported associated with concomitant alterations in suspected SQTS cases but comprehensive analyses conclude a benign role of the reported variant; therefore, it not should be included in the recommended analysis. Similar situation occurs with rare variant in the SCN5A gene; not conclusive diagnosis is established so no definite association gene-disease is currently reported. The same situation occurs with $S L C 4 A 3$ and its association with SQTS; no conclusive gene-disease relation has been reported so far. Hence, this new candidate gene should not be included in a comprehensive genetic analysis of patients with a conclusive diagnosis of SQTS. Therefore, considering all data reported so far, we believe that analysis of three genes (KCNH2, KCNQ1, and KCNJ2) should be performed in all suspected cases of SQTS, followed by a comprehensive genetic interpretation of each rare variant identified before translation into clinical practice. Gene-disease association is an important point to consider before clinical translation and, in concordance to current available data concerning other genes (CACNA1C, CACNA2D1, CACNB2b, SCN5A, and SLC4A3), they should not be analyzed in SQTS cases. Misinterpretation of the diagnosis is a current problem on SQTS. Therefore, several rare variants have been reported in families showing border QTc or Brugada-like ECG with short QT intervals, but not a certain SQTS. Performing an accurate clinical assessment in order to acquire (or not) a conclusive SQTS diagnosis of patients is crucial before genetic analysis but also in adoption of personalized suitable measures for prevention of SCD.

\subsection{Variants Reported as Associated with Short QT Syndrome}

Our comprehensive analysis of the only known CACNA2D1 variant, p.(Ser755Thr), revealed a benign role. This classification is due to conflicting data concerning phenotypes because it is associated with a BrS-like phenotype with concomitant shorter QT intervals. In $\mathrm{KCNH}$, nine variants are currently reported as DM, but data concerning global frequency along with in silico and functional analyses confirm a definitively pathogenic role of only four variants: p.(Asn588Lys) c.(1764C $>$ A), p.(Asn588Lys) c.(1764C>G), p.(Thr618Ile), and p.(Ile560Thr). Three variants, p.(Glu50Asp), p.(Ser631Ala) and p.(Trp927Gly), were classified as Likely Pathogenic due to a lack of functional data as well as family segregation. Another two variants in KCNH2, p.(Arg1135His) and p.(Arg164Cys), remain of ambiguous significance because of both were identified in patients showing BrS and shortened QT intervals, but did not result in a definite clinical diagnosis of SQTS. Of the three variants in KCNJ2 associated with SQTS so far, all three variants -p.(Met301Lys), p.(Glu299Val) and p.(Asp172Asn)- are classified definitively as Pathogenic, and all current published data support a deleterious role in SQTS. In KCNQ1, one variant, p.(Val307Leu), remains classified as Pathogenic and the other variant, p.(Phe279Ile), is currently classified as Likely Pathogenic. Finally, the potential pathogenic variant p.(Arg370His) reported in the SLC4A3 gene supposes the potential association of a new gene with SQTS but also represents a previously unappreciated mechanism for the development of the malignant arrhythmia. Notably, all carriers had a QTc $\leq 370 \mathrm{~ms}$ (not $340 \mathrm{~ms}$, accordingly to current clinical recommendations) supporting the no conclusive gene-disease association. In concordance to this no-conclusive association, functional studies were performed in zebrafish model; therefore, further studies should be performed in order to conclude a definite association.

It is important to remark that despite pathogenic role reported for some rare variants, clinical translation should be done with caution and personalized genetic interpretation is necessary; this fact is especially essential for rare variants without conclusive familial segregation. 


\subsection{Variants Reported as Potentially Associated with Short QT Syndrome}

In all consulted databases, sixteen variants in five genes were potentially associated with SQTS but had inconclusive data to make a final determination. Except for one, KCNQ1_p.(Val141Met), we recommend that these variants remain designated as having no conclusive relation to SQTS, though they may be implicated in phenotype modulation.

All eleven variants in CACNA1C and CACNB2 have been associated with BrS and shorter QT intervals, but their roles in SQTS remain ambiguous (classified as VUS) due to lack of conclusive clinical data. No patient carrying a variant in either calcium gene has been associated with a conclusive diagnose of SQTS. In addition, the variant CACNA1C_p.(Gly490Arg) has been identified in both LQTS and $\mathrm{BrS}$ cohorts. To our knowledge, nowadays there are no reports showing that a sole pathogenic variant cause two different pathologies in any human disease.

Concerning potassium genes, only one variant in KCNJ2_p.(LysK346Thr) had been reported as potentially causal in SQTS but published data reveal an ambiguous role. This is mainly due to a characteristic phenotype of autism and epilepsy with a concomitant short QT interval [39]. Although there is no conclusive association with SQTS, the authors of that previous study suggest a neuropsychiatric evaluation in patients with short QT intervals who carry variants in this potassium gene. The p.(Val141Met) variant in KCNQ1 was associated with a novel entity characterized by SQTS but concomitant with other clinical alterations. Curiously, HGMD classifies the variant as DM associated with atrial fibrillation, but no known current studies support this association. Due to a large number of reported cases associating this variant with SQTS, it is classified as definitively Pathogenic in SQTS, playing a deleterious role in clinical practice. The p.(Ile274Val) variant in KCNQ1 was identified in a SIDS cohort. Due to difficult characterization of death in SIDS, and a lack of functional evidence as well as family segregation, prudence should be taking into consideration before clinical translation [55]. Therefore, it remains classified as being of uncertain significance in SQTS. Finally, the p.(Arg259His) variant in KCNQ1 remains deleterious in LQTS due to identification in cohorts experiencing this arrhythmogenic disease. However, a solitary report identified a SQTS patient carrying this same variant [56]. Considering the reported contradictory data concerning clinical phenotype, this variant should be classified as VUS in both arrhythmogenic entities.

Finally, one variant of ambiguous significance in SQTS is reported in SCN5A, a gene associated with BrS but also responsible for nearly 10\% of LQTS cases [57]. The p.(Arg689His) variant has been identified in reports analyzing both LQTS and BrS patients. The same variant was identified in a case with Brugada-like ECG and short QT intervals. To our knowledge, there are no existing descriptions of a sole variant causing two different pathologies, as already mentioned. Considering all published data, this rare variant is classified as VUS for SQTS phenotypes.

A limitation of this study is the lack of reported information for some analyzed variants concerning functional studies as well as familial segregation, which are crucial pieces in the final translation into the clinical setting. It is important to remark that all predictions were performed according to current published data, but additional data may be available in the future. ACMG/AMP classification incorporates a vast number of elements to establish pathogenicity of the variant, despite the lack of validation in large study cohorts. Our classification was designed to have general applicability but also requires further functional studies for gene variant screenings to be correctly applied.

\section{Conclusions}

In conclusion, a limited number of SQTS variants have been classified as potentially pathogenic despite a lack of comprehensive classification following recent ACMG/AMP recommendations. Our comprehensive analysis of all published data for these variants revealed that only $28.12 \%$ of reported variants have a conclusive lethal role in SQTS. Importantly, variants with a definite deleterious role are located only in genes encoding potassium channels (KCNQ1, KCNH2, and KCNJ2). Rare variants located in genes encoding calcium and sodium channels are associated with the concomitant phenotype of BrS and slightly reduced QT interval but not an explicit diagnosis of SQTS. We recommend that care 
be taken with all previously reported variants classified as pathogenic and that the potassium channel variants be the only genetic clues used to diagnose SQTS. An exhaustive re-analysis is necessary to clarify the actual role of each variant related to SQTS before clinical translation is possible.

Author Contributions: Conceptualization, O.C., G.S.-B., J.B. and R.B.; methodology, O.C., A.F.-F., X.L. and S.C.; software, J.M., Bd.O., M.C. and C.F.-C.; validation, M.C., L.L., F.P. and M.P.; formal analysis, A.F.-F., X.L., S.C. and E.A.; investigation, S.C., E.A., P.J. and A.P.-S.; data curation, A.P.-S., L.L., F.P. and M.P.; writing-original draft preparation, O.C., A.F.-F., X.L.; writing-review and editing, all authors; supervision, J.B., R.B.; project administration, A.I., V.F.; funding acquisition, O.C. and R.B.

Funding: This work was supported by Obra Social "La Caixa Foundation" (ID 100010434), Fondo Investigacion Sanitaria -FIS PI14/01773 and PI17/01690- from the Instituto de Salud Carlos III (ISCIII), and Fundació Daniel Bravo Andreu. The CIBERCV is an initiative of the ISCIII, Spanish Ministry of Economy and Competitiveness (Fondos FEDER).

Conflicts of Interest: All authors declare no conflicts of interest to disclose.

\section{References}

1. Gussak, I.; Brugada, P.; Brugada, J.; Wright, R.S.; Kopecky, S.L.; Chaitman, B.R.; Bjerregaard, P. Idiopathic Short QT Interval: A New Clinical Syndrome? Cardiology 2000, 94, 99-102. [CrossRef]

2. Gollob, M.H.; Redpath, C.J.; Roberts, J.D. The short QT syndrome: Proposed diagnostic criteria. J. Am. Coll. Cardiol. 2011, 57, 802-812. [CrossRef]

3. Priori, S.G.; Wilde, A.A.; Horie, M.; Cho, Y.; Behr, E.R.; Berul, C.; Blom, N.; Brugada, J.; Chiang, C.E.; Huikuri, H.; et al. HRS/EHRA/APHRS expert consensus statement on the diagnosis and management of patients with inherited primary arrhythmia syndromes: Document endorsed by HRS, EHRA, and APHRS in May 2013 and by ACCF, AHA, PACES, and AEPC in June 2013. Heart Rhythm 2013, 10, 1932-1963. [CrossRef]

4. Priori, S.G.; Blomström-Lundqvist, C.; Mazzanti, A.; Blom, N.; Borggrefe, M.; Camm, J.; Elliott, P.M.; Fitzsimons, D.; Hatala, R.; Hindricks, G. 2015 ESC Guidelines for the management of patients with ventricular arrhythmias and the prevention of sudden cardiac death: The Task Force for the Management of Patients with Ventricular Arrhythmias and the Prevention of Sudden Cardiac Death of the European Society of Cardiology (ESC)Endorsed by: Association for European Paediatric and Congenital Cardiology (AEPC). Europace 2015, 17, 1601-1687.

5. El-Battrawy, I.; Schlentrich, K.; Besler, J.; Liebe, V.; Schimpf, R.; Lang, S.; EOdening, K.; Wolpert, C.; Zhou, X.; Borggrefe, M.; et al. Sex-differences in short QT syndrome: A systematic literature review and pooled analysis. Eur. J. Prev. Cardiol. 2019, 26. [CrossRef]

6. Bjerregaard, P. Diagnosis and management of short QT syndrome. Heart Rhythm 2018, 15, 1261-1267. [CrossRef]

7. El-Battrawy, I.; Besler, J.; Ansari, U.; Liebe, V.; Schimpf, R.; Tülümen, E.; Rudic, B.; Lang, S.; Odening, K.; Cyganek, L.; et al. Long-term follow-up of implantable cardioverter-defibrillators in Short QT syndrome. Clin. Res. Cardiol. 2019, 16, 1-7. [CrossRef]

8. Brugada, R.; Hong, K.; Dumaine, R.; Cordeiro, J.; Gaita, F.; Borggrefe, M.; Menendez, T.M.; Brugada, J.; Pollevick, G.D.; Wolpert, C.; et al. Sudden Death Associated With Short-QT Syndrome Linked to Mutations in HERG. Circulation 2004, 109, 30-35. [CrossRef]

9. Suzuki, H.; Hoshina, S.; Ozawa, J.; Sato, A.; Minamino, T.; Aizawa, Y.; Saitoh, A. Short QT syndrome in a boy diagnosed on screening for heart disease. Pediatr. Int. 2014, 56, 774-776. [CrossRef]

10. Richards, S.; Aziz, N.; Bale, S.; Bick, D.; Das, S.; Gastier-Foster, J.; Grody, W.W.; Hegde, M.; Lyon, E.; Spector, E.; et al. Standards and guidelines for the interpretation of sequence variants: A joint consensus recommendation of the American College of Medical Genetics and Genomics and the Association for Molecular Pathology. Genet. Med. 2015, 17, 405-423. [CrossRef]

11. Lek, M.; Exome Aggregation Consortium; Karczewski, K.J.; Minikel, E.V.; Samocha, K.E.; Banks, E.; Fennell, T.; O'Donnell-Luria, A.H.; Ware, J.S.; Hill, A.J.; et al. Analysis of protein-coding genetic variation in 60,706 humans. Nature 2016, 536, 285-291. [CrossRef]

12. Kobayashi, Y.; Yang, S.; Nykamp, K.; Garcia, J.; Lincoln, S.E.; Topper, S.E. Pathogenic variant burden in the ExAC database: An empirical approach to evaluating population data for clinical variant interpretation. Genome Med. 2017, 9, 13. [CrossRef] 
13. Tayoun, A.N.A.; Pesaran, T.; Distefano, M.T.; Oza, A.; Rehm, H.L.; Biesecker, L.G.; Harrison, S.M.; ClinGen Sequence Variant Interpretation Working Group (ClinGen SVI). Recommendations for interpreting the loss of function PVS1 ACMG/AMP variant criterion. Hum. Mutat. 2018, 39, 1517-1524. [CrossRef]

14. Nykamp, K.; The Invitae Clinical Genomics Group; Anderson, M.; Powers, M.; Garcia, J.; Herrera, B.; Ho, Y.-Y.; Kobayashi, Y.; Patil, N.; Thusberg, J.; et al. Sherloc: A comprehensive refinement of the ACMG-AMP variant classification criteria. Genet. Med. 2017, 19, 1105-1117. [CrossRef]

15. Templin, C.; Ghadri, J.R.; Rougier, J.S.; Baumer, A.; Kaplan, V.; Albesa, M.; Sticht, H.; Rauch, A.; Puleo, C.; $\mathrm{Hu}, \mathrm{D}$; ; et al. Identification of a novel loss-of-function calcium channel gene mutation in short QT syndrome (SQTS6). Eur. Heart J. 2011, 32, 1077-1088. [CrossRef]

16. Antzelevitch, C.; Pollevick, G.D.; Cordeiro, J.M.; Casis, O.; Sanguinetti, M.C.; Aizawa, Y.; Guerchicoff, A.; Pfeiffer, R.; Oliva, A.; Wollnik, B.; et al. Loss-of-Function Mutations in the Cardiac Calcium Channel Underlie a New Clinical Entity Characterized by ST-Segment Elevation, Short QT Intervals, and Sudden Cardiac Death. Circulation 2007, 115, 442-449. [CrossRef]

17. Simms, B.A.; Zamponi, G.W. The Brugada syndrome mutation A39V does not affect surface expression of neuronal rat Cav1.2 channels. Mol. Brain 2012, 5, 9. [CrossRef]

18. Burashnikov, E.; Pfeiffer, R.; Barajas-Martínez, H.; Delpón, E.; Hu, D.; Desai, M.; Borggrefe, M.; Haissaguerre, M.; Kanter, R.; Pollevick, G.D.; et al. Mutations in the cardiac L-type calcium channel associated with inherited J-wave syndromes and sudden cardiac death. Heart Rhythm 2010, 7, 1872-1882. [CrossRef]

19. Ye, D.; Tester, D.J.; Zhou, W.; Papagiannis, J.; Ackerman, M.J. A pore-localizing CACNA1C-E1115K missense mutation, identified in a patient with idiopathic QT prolongation, bradycardia, and autism spectrum disorder, converts the L-type calcium channel into a hybrid nonselective monovalent cation channel. Heart Rhythm 2019, 16, 270-278. [CrossRef]

20. Fukuyama, M.; Ohno, S.; Wang, Q.; Kimura, H.; Makiyama, T.; Itoh, H.; Ito, M.; Horie, M. L-Type Calcium Channel Mutations in Japanese Patients With Inherited Arrhythmias. Circ. J. 2013, 77, 1799-1806. [CrossRef]

21. Fukuyama, M.; Ohno, S.; Wang, Q.; Shirayama, T.; Itoh, H.; Horie, M. Nonsense-mediated mRNA decay due to a CACNA1C splicing mutation in a patient with Brugada syndrome. Heart Rhythm 2014, 11, 629-634. [CrossRef]

22. Gaita, F.; Giustetto, C.; Bianchi, F.; Wolpert, C.; Schimpf, R.; Riccardi, R.; Grossi, S.; Richiardi, E.; Borggrefe, M. Short QT Syndrome: A familial cause of sudden death. Circulation 2003, 108, 965-970. [CrossRef]

23. Whittaker, D.G.; Hancox, J.; Zhang, H. In silico Assessment of Pharmacotherapy for Human Atrial Patho-Electrophysiology Associated With hERG-Linked Short QT Syndrome. Front. Physiol. 2018, 9, 1888. [CrossRef]

24. Zhao, Z.; Li, X.; El-Battrawy, I.; Lan, H.; Zhong, R.; Xu, Q.; Huang, M.; Liao, Z.; Lang, S.; Zimmermann, W.; et al. Drug Testing in Human-Induced Pluripotent Stem Cell-Derived Cardiomyocytes From a Patient With Short QT Syndrome Type 1. Clin. Pharmacol. Ther. 2019, 4. [CrossRef]

25. El-Battrawy, I.; Lan, H.; Cyganek, L.; Zhao, Z.; Li, X.; Buljubasic, F.; Lang, S.; Yücel, G.; Sattler, K.; Zimmermann, W.; et al. Modeling Short QT Syndrome Using Human-Induced Pluripotent Stem Cell-Derived Cardiomyocytes. J. Am. Heart Assoc. 2018, 7, e007394. [CrossRef]

26. Itoh, H.; Sakaguchi, T.; Ashihara, T.; Ding, W.-G.; Nagaoka, I.; Oka, Y.; Nakazawa, Y.; Yao, T.; Jo, H.; Ito, M.; et al. A novel KCNH2 mutation as a modifier for short QT interval. Int. J. Cardiol. 2009, 137, 83-85. [CrossRef]

27. Redpath, C.J.; Green, M.S.; Birnie, D.H.; Gollob, M.H. Rapid genetic testing facilitating the diagnosis of short QT syndrome. Can. J. Cardiol. 2009, 25, e133-e135. [CrossRef]

28. Sun, Y.; Quan, X.-Q.; Fromme, S.; Cox, R.H.; Zhang, P.; Zhang, L.; Guo, D.; Guo, J.; Patel, C.; Kowey, P.R.; et al. A novel mutation in the KCNH2 gene associated with short QT syndrome. J. Mol. Cell. Cardiol. 2011, 50, 433-441. [CrossRef]

29. Hu, D.; Li, Y.; Zhang, J.; Pfeiffer, R.; Gollob, M.H.; Healey, J.; Harrell, D.T.; Makita, N.; Abe, H.; Sun, Y.; et al. The Phenotypic Spectrum of a Mutation Hotspot Responsible for the Short QT Syndrome. JACC Clin. Electrophysiol. 2017, 3, 727-743. [CrossRef]

30. Guo, F.; Sun, Y.; Wang, X.; Wang, H.; Wang, J.; Gong, T.; Chen, X.; Zhang, P.; Su, L.; Fu, G.; et al. Patient-Specific and Gene-Corrected Induced Pluripotent Stem Cell-Derived Cardiomyocytes Elucidate Single-Cell Phenotype of Short QT Syndrome. Circ. Res. 2019, 124, 66-78. [CrossRef] 
31. Wang, Q.; Ohno, S.; Ding, W.G.; Fukuyama, M.; Miyamoto, A.; Itoh, H.; Makiyama, T.; Wu, J.; Bai, J.; Hasegawa, K.; et al. Gain-of-Function KCNH2 Mutations in Patients with Brugada Syndrome. J. Cardiovasc. Electrophysiol. 2014, 25, 522-530. [CrossRef]

32. Butler, A.; Zhang, Y.; Stuart, A.G.; Dempsey, C.E.; Hancox, J.C. Functional and pharmacological characterization of an S5 domain hERG mutation associated with short QT syndrome. Heliyon 2019, 5, e01429. [CrossRef]

33. Akdis, D.; Saguner, A.M.; Medeiros-Domingo, A.; Schaller, A.; Balmer, C.; Steffel, J.; Brunckhorst, C.; Duru, F. Multiple clinical profiles of families with the short QT syndrome. Europace 2018, 20, f113-f121. [CrossRef]

34. Butler, A.; Zhang, Y.; Stuart, A.G.; Dempsey, C.E.; Hancox, J.C. Action potential clamp characterization of the S631A hERG mutation associated with short QT syndrome. Physiol. Rep. 2018, 6, e13845. [CrossRef]

35. Priori, S.G.; Pandit, S.V.; Rivolta, I.; Berenfeld, O.; Ronchetti, E.; Dhamoon, A.; Napolitano, C.; Anumonwo, J.; Di Barletta, M.R.; Gudapakkam, S.; et al. A Novel Form of Short QT Syndrome (SQT3) Is Caused by a Mutation in the KCNJ2 Gene. Circ. Res. 2005, 96, 800-807. [CrossRef]

36. Hattori, T.; Makiyama, T.; Akao, M.; Ehara, E.; Ohno, S.; Iguchi, M.; Nishio, Y.; Sasaki, K.; Itoh, H.; Yokode, M.; et al. A novel gain-of-function KCNJ2 mutation associated with short-QT syndrome impairs inward rectification of Kir2.1 currents. Cardiovasc. Res. 2012, 93, 666-673. [CrossRef]

37. Hasegawa, K.; Ashihara, T.; Kimura, H.; Jo, H.; Itoh, H.; Yamamoto, T.; Aizawa, Y.; Horie, M. Long-term Pharmacological Therapy of Brugada Syndrome: Is J-wave Attenuation a Marker of Drug Efficacy? Intern. Med. 2014, 53, 1523-1526. [CrossRef]

38. Deo, M.; Ruan, Y.; Pandit, S.V.; Shah, K.; Berenfeld, O.; Blaufox, A.; Cerrone, M.; Noujaim, S.F.; Denegri, M.; Jalife, J.; et al. KCNJ2 mutation in short QT syndrome 3 results in atrial fibrillation and ventricular proarrhythmia. Proc. Natl. Acad. Sci. USA 2013, 110, 4291-4296. [CrossRef]

39. Ambrosini, E.; Sicca, F.; Napolitano, C.; Servettini, I.; Moro, F.; Ruan, Y.; Guglielmi, L.; Pieroni, S.; Servillo, G.; Lanciotti, A.; et al. Genetically induced dysfunctions of Kir2.1 channels: Implications for short QT3 syndrome and autism-epilepsy phenotype. Hum. Mol. Genet. 2014, 23, 4875-4886. [CrossRef]

40. Bellocq, C.; Van Ginneken, A.C.; Bezzina, C.R.; Alders, M.; Escande, D.; Mannens, M.M.; Baró, I.; Wilde, A.A. Mutation in the KCNQ1 Gene Leading to the Short QT-Interval Syndrome. Circulation 2004, 109, 2394-2397. [CrossRef]

41. Seebohm, G.; Scherer, C.R.; Busch, A.E.; Lerche, C. Identification of Specific Pore Residues Mediating KCNQ1 Inactivation. J. Boil. Chem. 2001, 276, 13600-13605. [CrossRef] [PubMed]

42. Hong, K.; Bjerregaard, P.; Gussak, I.; Brugada, R. Short QT Syndrome and Atrial Fibrillation Caused by Mutation in KCNH2. J. Cardiovasc. Electrophysiol. 2005, 16, 394-396. [CrossRef] [PubMed]

43. Campbell, C.M.; Campbell, J.D.; Thompson, C.H.; Galimberti, E.S.; Darbar, D.; Vanoye, C.G.; George, A.L. Selective Targeting of Gain-of-function KCNQ1 Mutations Predisposing to Atrial Fibrillation. Circ. Arrhythmia Electrophysiol. 2013, 6, 960-966. [CrossRef] [PubMed]

44. Fischbach, P.; Gebauer, R.; Villafañe, J. Short QT Syndrome Manifesting with Neonatal Atrial Fibrillation and Bradycardia. Cardiology 2014, 128, 236-240.

45. Sarquella-Brugada, G.; Campuzano, O.; Iglesias, A.; Grueso, J.; Bradley, D.J.; Kerst, G.; Shmorhun, D.; Brugada, J.; Brugada, R. Short QT and atrial fibrillation: A KCNQ1 mutation-specific disease. Late follow-up in three unrelated children. HeartRhythm Case Rep. 2015, 1, 193-197. [CrossRef] [PubMed]

46. Arnestad, M.; Crotti, L.; Rognum, T.O.; Insolia, R.; Pedrazzini, M.; Ferrandi, C.; Vege, A.; Wang, D.W.; Rhodes, T.E.; George, A.L.; et al. Prevalence of Long-QT Syndrome Gene Variants in Sudden Infant Death Syndrome. Circulation 2007, 115, 361-367. [CrossRef]

47. Millat, G.; Chevalier, P.; Da Costa, A.; Bouvagnet, P.; Kugener, B.; Fayol, L.; Armengod, C.G.; Oddou, B.; Chanavat, V.; Froidefond, E.; et al. Spectrum of pathogenic mutations and associated polymorphisms in a cohort of 44 unrelated patients with long QT syndrome. Clin. Genet. 2006, 70, 214-227. [CrossRef] [PubMed]

48. Mazzanti, A.; Kanthan, A.; Monteforte, N.; Memmi, M.; Bloise, R.; Novelli, V.; Miceli, C.; O’Rourke, S.; Borio, G.; Zienciuk-Krajka, A.; et al. Novel Insight Into the Natural History of Short QT Syndrome. J. Am. Coll. Cardiol. 2014, 63, 1300-1308. [CrossRef]

49. Ackerman, M.J.; Splawski, I.; Makielski, J.C.; Tester, D.J.; Will, M.L.; Timothy, K.W.; Keating, M.T.; Jones, G.; Chadha, M.; Burrow, C.R.; et al. Spectrum and prevalence of cardiac sodium channel variants among black, white, Asian, and Hispanic individuals: Implications for arrhythmogenic susceptibility and Brugada/long QT syndrome genetic testing. Heart Rhythm 2004, 1, 600-607. [CrossRef] 
50. Napolitano, C.; Priori, S.G.; Schwartz, P.J.; Bloise, R.; Ronchetti, E.; Nastoli, J.; Bottelli, G.; Cerrone, M.; Leonardi, S. Genetic testing in the long QT syndrome: Development and validation of an efficient approach to genotyping in clinical practice. JAMA 2005, 294, 2975-2980. [CrossRef]

51. Nakajima, T.; Kaneko, Y.; Saito, A.; Irie, T.; Tange, S.; Iso, T.; Kurabayashi, M. Identification of Six Novel SCN5A Mutations in Japanese Patients With Brugada Syndrome. Int. Heart J. 2011, 52, 27-31. [CrossRef] [PubMed]

52. Hu, J.; Yu, J.; Cheng, X.S.; Brugada, R.; Hong, K. Concomitant brugada-like and short qt electrocardiogram linked to scn5a mutation. Heart 2012, 98, E315.3-E316.

53. Thorsen, K.; Dam, V.S.; Kjaer-Sorensen, K.; Pedersen, L.N.; Skeberdis, V.A.; Jurevicius, J.; Treinys, R.; Petersen, I.M.B.S.; Nielsen, M.S.; Oxvig, C.; et al. Loss-of-activity-mutation in the cardiac chloride-bicarbonate exchanger AE3 causes short QT syndrome. Nat. Commun. 2017, 8, 1696. [CrossRef] [PubMed]

54. Riuró, H.; Campuzano, O.; Berne, P.; Arbelo, E.; Iglesias, A.; Pérez-Serra, A.; Coll-Vidal, M.; Partemi, S.; Mademont-Soler, I.; Picó, F.; et al. Genetic analysis, in silico prediction, and family segregation in long QT syndrome. Eur. J. Hum. Genet. 2015, 23, 79-85. [CrossRef] [PubMed]

55. Sarquella-Brugada, G.; Campuzano, O.; Cesar, S.; Iglesias, A.; Fernández, A.; Brugada, J.; Brugada, R. Sudden infant death syndrome caused by cardiac arrhythmias: Only a matter of genes encoding ion channels? Int. J. Leg. Med. 2016, 130, 415-420. [CrossRef] [PubMed]

56. Wu, Z.J.; Huang, Y.; Fu, Y.C.; Zhao, X.J.; Zhu, C.; Zhang, Y.; Xu, B.; Zhu, Q.L.; Li, Y. Characterization of a Chinese KCNQ1 mutation (R259H) that shortens repolarization and causes short QT syndrome 2. J. Geriatr. Cardiol. 2015, 12, 394-401. [PubMed]

57. Fernández-Falgueras, A.; Sarquella-Brugada, G.; Brugada, J.; Brugada, R.; Campuzano, O. Cardiac Channelopathies and Sudden Death: Recent Clinical and Genetic Advances. Biology 2017, 6, 7. [CrossRef]

(C) 2019 by the authors. Licensee MDPI, Basel, Switzerland. This article is an open access article distributed under the terms and conditions of the Creative Commons Attribution (CC BY) license (http://creativecommons.org/licenses/by/4.0/). 